\title{
Infinite Measures on von Neumann Algebras
}

\author{
Stanisław Goldstein • Adam Paszkiewicz
}

Received: 26 October 2014 / Accepted: 19 January 2015 / Published online: 10 February 2015

(C) Springer Science+Business Media New York 2015

\begin{abstract}
Infinite measures on von Neumann algebras are classified according to properties analogous to those from classical measure theory. These properties are carefully examined in case of semifinite von Neumann algebras. Some examples are also given and the direction for further research indicated.
\end{abstract}

Keywords Infinite measure $\cdot$ Weight $\cdot$ Semifinite $\cdot$ Densely semifinite $\cdot$ Strictly semifinite $\cdot$ von Neumann algebra

\section{Introduction}

The aim of the paper is to look at weights on von Neumann algebras from the perspective of commutative measure theory, i.e. to treat them as infinite measures. Weights have an important place in the theory of operator algebras. For example, the flow of weights (see [9, Ch. XII, Section 4]) is an essential tool in the structural theory of type III von Neumann algebras. The most important class of weights are the semifinite ones, defined in a way adjusted to the noncommutative situation. In particular, the subalgebra on which the weight is finite cannot be defined as it is in the commutative situation. Moreover, the definition of semifiniteness cannot be translated to the noncommutative context in the most direct way. We try to see what classes of weights one obtains when applying the classical definitions concerned with infinite measures to the noncommutative situation.

The second author was supported by NCN grant no. 2011/01/B/ST1/03994

S. Goldstein $(\bowtie) \cdot$ A. Paszkiewicz

Faculty of Mathematics and Computer Science, Łódź University, Banacha 22, 90-238 Łódź, Poland

e-mail: goldstei@math.uni.lodz.pl

A. Paszkiewicz

e-mail: adampasz@math.uni.lodz.pl 
By translating the definition of semifiniteness we get a class of weights that we call densely semifinite. On the other hand, an important subclass of semifinite measures, namely the $\sigma$-finite ones, lead to the notion of orthogonally semifinite weight, with the provision that 'countable' is replaced by 'arbitrary' in the definition (compare Proposition 5). We also consider a well established (see [7, S.10.9], [9, Ch. VIII, Section 2, Ex.1]) class of strictly semifinite weights, introduced by Combes [2], that together with the integrable ones are used substantially in the theory of type III algebras. This paper is the first attempt at comparison of the various classes of weights. We concentrate on the semifinite case, since it allows us to use methods that are elementary in the sense of not using the modular theory in a substantial way. Our results are complete for the full algebra $\mathbb{B}(H)$, a case of special interest to physicists.

The proofs are deliberately a bit longer than they could have been, to make the paper accessible to the people with only secondary interest in operator algebras. We hope that the measure theoretic aspect of the paper might catch the attention of a wider audience. The authors would like to thank Ken Dykema for directing their attention to the paper of Herman and Takesaki [6], and the anonymous reviewers for their useful comments and corrections.

\section{Definitions}

Assume $\varphi$ is a normal faithful weight on a von Neumann algebra $M$ (in what follows, the assumption of faithfulness is not always used, but it does not hurt). We denote by $\operatorname{Proj} M$ the set of orthogonal projections of $M$, and by $\left\{\sigma_{t}^{\varphi}\right\}_{t \in \mathbb{R}}$ the modular automorphism group of $M$ with respect to $\varphi$.

We use the following standard notation:

$$
\begin{aligned}
\mathfrak{m}_{\varphi}^{+} & =\left\{x \in M_{+}: \varphi(x)<\infty\right\} \\
\mathfrak{n}_{\varphi} & =\left\{x \in M: x^{*} x \in \mathfrak{m}_{\varphi}^{+}\right\} \\
\mathfrak{m}_{\varphi} & =\mathfrak{n}_{\varphi}^{*} \mathfrak{n}_{\varphi}=\text { linear span of } \mathfrak{m}_{\varphi}^{+} \\
M_{\varphi} & =\left\{x \in M: \sigma_{t}^{\varphi}(x)=x \forall t \in \mathbb{R}\right\} \\
& =\left\{x \in M: x \mathfrak{m}_{\varphi} \subseteq \mathfrak{m}_{\varphi}, \mathfrak{m}_{\varphi} x \subseteq \mathfrak{m}_{\varphi} \text { and } \varphi(x y)=\varphi(y x) \forall y \in \mathfrak{m}_{\varphi}\right\}
\end{aligned}
$$

Let us recall (see [1] and [9, Ch. VII, Lemma 1.2]) that $\mathfrak{m}_{\varphi}^{+}$is a hereditary subcone of $M_{+}$, $\mathfrak{n}_{\varphi}$ is a left ideal of $M$, and $\mathfrak{m}_{\varphi}$ is a ${ }^{*}$-subalgebra of $M$ such that $\mathfrak{m}_{\varphi} \cap M_{+}=\mathfrak{m}_{\varphi}^{+}$. Moreover, $M_{\varphi}$ is a von Neumann subalgebra of $M$ called the centralizer of $\varphi$ in $M$.

Definition 1 We call the weight $\varphi$ :

(1) semifinite if $\mathfrak{m}_{\varphi}^{+}$generates $M$ as a von Neumann algebra (equivalently, the *-algebra $\mathfrak{m}_{\varphi}$ is dense in $M$ in any of the following topologies: weak, $\sigma$-weak, strong, $\sigma$-strong, strong*, $\sigma$-strong*);

(2) orthogonally semifinite if there exists a family $\left\{e_{i}\right\}$ such that $e_{i} \in \operatorname{Proj} M \cap \mathfrak{m}_{\varphi}^{+}$for all $i$, and $\sum e_{i}=1$; 
(3) densely semifinite if for each non-zero $e \in \operatorname{Proj} M$ there exists $f \in \operatorname{Proj} M$ such that $0 \neq f \leq e$ and $\varphi(f)<\infty$;

(4) strictly semifinite if there exists a family $\left\{\varphi_{i}\right\}$ of positive normal functionals from $\mathrm{M}$ (i.e. $\varphi_{i} \in M_{*}^{+}$for all $i$ ) with pairwise orthogonal supports such that $\sum \operatorname{supp} \varphi_{i}=1$ and $\varphi=\sum \varphi_{i}$ (pointwise). Equivalently, $\varphi$ is strictly semifinite if its restriction to the centralizer $M_{\varphi}$ is semifinite. (See [2, 3], [7, S.10.9], [9, Ch. VIII, Section 2, Ex.1]).

\section{General algebras}

Let us start with the comparison of the notions in the most general case.

Proposition 2 (1) Every strictly semifinite weight is orthogonally semifinite.

(2) A weight is densely semifinite if and only if it is orthogonally semifinite on each reduced von Neumann algebra eMe, where e $\in$ ProjM. In particular, every densely semifinite weight is orthogonally semifinite.

(3) Every orthogonally semifinite weight is semifinite.

Proof (1) Put $e_{i}=\operatorname{supp} \varphi_{i}$.

(2) “ $\Rightarrow$ " Let $\left\{e_{i}\right\}$ be a maximal family of mutually orthogonal non-zero subprojections of $e$ of finite weight (use Zorn lemma and dense semifiniteness to show its existence). If $\sum e_{i}<e$, then we could enlarge the family by adding to it a non-zero projection $\leq e-$ $\sum e_{i}$, hence $\sum e_{i}=e$ and $\varphi \mid e M e$ is orthogonally semifinite. " $\Leftarrow$ " Let $0 \neq e \in \operatorname{Proj} M$. If $\varphi$ is orthogonally semifinite on $e M e$, then $e=\sum e_{i}$ with $e_{i} \in \operatorname{Proj} M, \varphi\left(e_{i}\right)<\infty$, and at least one of the $e_{i}$ 's must be non-zero.

(3) Assume $M$ acts on a Hilbert space $H$, and $\left\{e_{i}\right\}_{i \in I}$ is the family from the definition. For $J \subseteq I, J$ finite, put $f_{J}=\sum_{i \in J} e_{i}$. We are going to show that $x \in M, f_{J} x f_{J} \in \mathfrak{m}_{\varphi}$ and $f_{J} x f_{J} \rightarrow x$ strongly, which shows the semifiniteness of $\varphi$. In fact, for any $i, j$ we have $e_{i}, e_{j} \in \mathfrak{n}_{\varphi} \cap \mathfrak{n}_{\varphi}^{*}$ and $\mathfrak{n}_{\varphi}$ is a left ideal, so that $e_{i} x e_{j} \in \mathfrak{n}_{\varphi}^{*} \mathfrak{n}_{\varphi}=\mathfrak{m}_{\varphi}$. Now, $f_{J} \rightarrow 1$ strongly and, for any $\xi \in H$,

$$
\begin{aligned}
\left\|\left(f_{J} x f_{J}-x\right) \xi\right\| & \leq\left\|f_{J} x\left(1-f_{J}\right) \xi\right\|+\left\|\left(1-f_{J}\right) x \xi\right\| \\
& \leq\|x\|\left\|\left(1-f_{J}\right) \xi\right\|+\left\|\left(1-f_{J}\right) x \xi\right\| \rightarrow 0,
\end{aligned}
$$

which ends the proof.

\section{Traces}

It is easy to see that the four notions of semifiniteness given above coincide in the commutative case. We show that in fact it is enough for the measure to be 'commutative'.

Proposition 3 Let $\varphi=\tau$ be a faithful normal trace on a von Neumann algebra M. Then conditions (1), (2), (3), (4) from Definition 1 are equivalent.

Proof We know from Proposition 2 that (3) $\Rightarrow$ (2), (4) $\Rightarrow$ (2) and (2) $\Rightarrow$ (1).

(1) $\Rightarrow$ (3). Let $e \in \operatorname{Proj} M$. By the Kaplansky density theorem [8, Ch. II, §4], there is a net $\left(x_{i}\right), x_{i} \in M_{+},\left\|x_{i}\right\| \leq 1$ such that $x_{i} \rightarrow e$ strongly and $\tau\left(x_{i}\right)<\infty$ for all $i$. Then $\tau\left(e x_{i} e\right)=\tau\left(x_{i}^{1 / 2} e x_{i}^{1 / 2}\right) \leq \tau\left(x_{i}\right)<\infty$ for all $i$. Since $e x_{i} e \rightarrow e$ strongly, there must exist 
an $i_{0}$ such that $e x_{i_{0}} e \neq 0$. Hence, for some $\epsilon>0,0 \neq \epsilon \chi_{[\epsilon, \infty[}\left(e x_{i_{0}} e\right) \leq e x_{i_{0}} e \leq e$. Put $f=\chi_{[\epsilon, \infty[}\left(e x_{i_{0}} e\right)$. Then $0 \neq f \leq(1 / \epsilon) e$, so that $f \leq e$ and $\tau(f)<\infty$.

$(2) \Rightarrow(4)$. Take $\left\{e_{i}\right\}$ with $\tau\left(e_{i}\right)<\infty$ and $\sum e_{i}=1$. Then, for all $i, j, e_{j} x e_{i} \in \mathfrak{m}_{\varphi}$ (cf. proof of part (3) of Proposition 2) and $\tau(x)=\sup _{n} \sum_{i, j}^{n} \tau\left(e_{j} x e_{i}\right)=\sum_{i} \tau\left(e_{i} x e_{i}\right)$. Put $\tau_{i}=e_{i} \tau e_{i}$ (where $e \tau e$ means $\tau(e \cdot e)$ ). Then $\operatorname{supp} \tau_{i}=e_{i}$ and $\tau=\sum \tau_{i}$.

\section{$5 \sigma$-finite algebras}

In the classical measure theory we usually assume a measure to be $\sigma$-finite. There is a natural way of defining such a measure in the noncommutative measure theory:

Definition 4 A (faithful normal) weight on $M$ is $\sigma$-finite if there is a sequence $\left\{e_{n}\right\}$ of mutually orthogonal projections from $M$ with $\sum_{n=1}^{\infty} e_{n}=1$ such that $\varphi\left(e_{n}\right)<\infty$ for all $n$.

It turns our that the definition is, in fact, not needed - the proper notion, namely that of an orthogonally semifinite weight, has already been defined. This follows from the proposition below.

Proposition 5 (1) If $\varphi$ is $\sigma$-finite, then it is orthogonally semifinite.

(2) If $\varphi$ is orthogonally semifinite and the algebra $M$ is $\sigma$-finite, then $\varphi$ is $\sigma$-finite.

(3) There are no (faithful normal) $\sigma$-finite weights on a non- $\sigma$-finite von Neumann algebra.

Proof (1) This is trivial.

(2) Let $\left\{e_{i}\right\}$ be a family of projections from the definition of orthogonal semifiniteness. Since it is an orthogonal family and the algebra is $\sigma$-finite, all but a countable number of the projections must be zero.

(3) Let $\left\{f_{i}\right\}_{i \in I}$ such that for all $i \in I, 0 \neq f_{i} \in \operatorname{Proj} M, \sum_{i \in I} f_{i}=1$ and $\operatorname{card} I>\aleph_{0}$. Suppose that there is a countable family $\left\{e_{n}\right\}$ of mutually orthogonal projections from $M$ such that $\varphi\left(e_{n}\right)<\infty$ for all $n$ and $\sum e_{n}=1$. As in the proof of part (3) of Proposition $2, e_{n} f_{i} e_{n} \in \mathfrak{m}_{\varphi}$ for any $i, n$. Since $\sum_{i \in I} \varphi\left(e_{n} f_{i} e_{n}\right)=\varphi\left(e_{n}\left(\sum_{i \in I} f_{i}\right) e_{n}\right)=$ $\varphi\left(e_{n}\right)<\infty$, for each $n$ there is a set $I_{n}$ such that $\operatorname{card} I \backslash I_{n} \leq \aleph_{0}$ and $\varphi\left(e_{n} f_{i} e_{n}\right)=0$ for $i \in I_{n}$. Thus the intersection of $I_{n}$ 's is non-empty and there is $i_{0} \in I$ such that $\varphi\left(e_{n} f_{i_{0}} e_{n}\right)=0$ for all $n$. The faithfulness of $\varphi$ implies $\left(f_{i_{0}} e_{n}\right)^{*}\left(f_{i_{0}} e_{n}\right)=$ 0 , hence $f_{i_{0}} e_{n} f_{i_{0}}=0$ for all $n$. Summing over $n$ gives $f_{i_{0}}=0$, which is a contradiction.

\section{Semifinite algebras}

Here we gather the properties of weights that hold in all semifinite von Neumann algebras. Let $M$ be a semifinite von Neumann algebra with a faithful normal semifinite (f.n.s.) trace $\tau$, and let $\varphi$ be a faithful normal weight on $M$. We denote by $h$ the density of $\varphi$ with respect to $\tau$ (for existence, see [9, Ch. VIII, Thm. 3.14]), i.e. $\varphi(\cdot)=\tau(h \cdot)$ in the sense explained in [9, Ch. VIII, Section 2, Lemma 2.7], . Recall that $h$ is $\tau$-measurable if, for some $n \in \mathbb{N}$, $\tau\left(\chi_{[n, \infty[}(h)\right)<\infty$. In the sequel, we say that the positive operator $h$ has pure point spectrum if there exists an orthogonal family $\left\{e_{i}\right\}$ of projections from $M$ and a family of positive 
numbers $\left\{\lambda_{i}\right\}$ such that $h=\sum \lambda_{i} e_{i}$. It is clear that the spectrum of $h$ is in this case the closure of the set of eigenvalues of $h$.

Theorem 6 With the assumptions above, we have:

(1) If $\varphi$ is semifinite, then it is orthogonally semifinite.

(2) If $h$ is $\tau$-measurable (for some f.n.s. trace on $M$ ), then $\varphi$ is densely semifinite.

(3) If h has pure point spectrum, then $\varphi$ is strictly semifinite.

Proof (1) Put $e_{n}=\chi_{[n, n+1[}(h)$. Since all the notions of semifiniteness coincide for a trace (Proposition 3), for each $n$ there is a family $\left\{f_{i}^{(n)}\right\}_{i \in I_{n}}$ such that for all $n$ and $i \in$ $I_{n}, \tau\left(f_{i}^{(n)}\right)<\infty$ and, for all $n, e_{n}=\sum_{i \in I_{n}} f_{i}^{(n)}$. Note that $\varphi\left(f_{i}^{(n)}\right)=\tau\left(h\left(f_{i}^{(n)}\right)\right) \leq$ $(n+1) \tau\left(f_{i}^{(n)}\right)<\infty$, which means that $\varphi$ is orthogonally semifinite.

(2) Assume $h$ is $\tau$-measurable. Let $0 \neq p \in \operatorname{Proj} M$. Since $\tau$ is densely semifinite (see Proposition 3), there is a non-zero projection $q \leq p$ with $\tau(q)<\infty$. Put $e_{n}=$ $\chi_{[0, n[}(h)$. Then $1-e_{n} \rightarrow 0$ strongly, and, from measurability of $h, \tau\left(1-e_{n}\right)<\infty$ for sufficiently large $n$, hence $\tau\left(1-e_{n}\right) \rightarrow 0$. Put $r_{n}=q \wedge e_{n}$. If $r_{n}=0$ then $q \prec 1-e_{n}$ (see [8, Ch.V, Prop. 1.6]), so that $\tau(q) \leq \tau\left(1-e_{n}\right)$. It follows that for some $n_{0}, r_{n_{0}} \neq 0$, otherwise $\tau(q)=0$, which gives $q=0$, a contradiction. Note that $r_{n_{0}} \leq q \leq p$ and

$$
\varphi\left(r_{n_{0}}\right)=\tau\left(h\left(q \wedge e_{n_{0}}\right)\right) \leq n_{0} \tau\left(q \wedge e_{n_{0}}\right) \leq n_{0} \tau(q)<\infty .
$$

(3) If $h$ has pure point spectrum, then $h=\sum \lambda_{i} e_{i}$, where $\lambda_{i}>0$ and $e_{i}$ form an orthogonal family of non-zero projections from $M$. Then $\varphi=\sum \varphi_{i}$, where $\varphi_{i}=\tau\left(\lambda_{i} e_{i} \cdot\right)$. By Proposition 3, for each $i$ the projection $e_{i}$ decomposes into an orthogonal sum of projections with finite trace, hence each $\varphi_{i}$ decomposes into a sum of positive normal functionals with mutually orthogonal supports. This shows that $\varphi$ is strictly semifinite.

\section{Finite algebras}

Let $M$ be a finite von Neumann algebra, and let $\varphi$ be a faithful normal weight on $M$. If $M$ is $\sigma$-finite, then there exists a faithful normal finite trace on $M$, and this is the trace that we use in this case, with $h$ being the density of $\varphi$ with respect to the trace.

Proposition 7 For a finite von Neumann algebra, all the notions from Definition 1 coincide.

Proof (1) Assume first that the algebra in question is $\sigma$-finite. We need to show that if $\varphi$ is semifinite, then it is both densely and strictly semifinite. Note that any positive densely defined operator affiliated with a finite $\sigma$-finite von Neumann algebra is measurable, hence the weight is densely semifinite by Theorem 6, (2). Put $e_{n}=\chi_{[n, n+1[}(h)$. Then $\sum_{n=0}^{\infty} e_{n}=1$, and $\varphi\left(e_{n}\right)=\tau\left(h e_{n}\right) \leq(n+1) \tau\left(e_{n}\right)<\infty$. Thus $e_{n} \varphi e_{n}$ are positive normal functionals on $M$ with mutually orthogonal supports. We shall show that $\varphi=$ $\sum e_{n} \varphi e_{n}$. First of all, for a fixed $x \in M_{+}, e_{n} x e_{n} \leq\|x\| e_{n}$, so that $e_{n} x e_{n} \in \mathfrak{m}_{\varphi}^{+}$. Hence $x^{1 / 2} e_{n} \in \mathfrak{n}_{\varphi}$ for all $n$, so that $e_{m} x e_{n} \in \mathfrak{m}_{\varphi}$ for all $m, n$. If $m \neq n$, 


$$
\begin{aligned}
\varphi\left(e_{m} x e_{n}\right) & =\tau\left(h^{1 / 2} e_{m} x e_{n} h^{1 / 2}\right)=\tau\left(x^{1 / 2} e_{n} h^{1 / 2} h^{1 / 2} e_{m} x^{1 / 2}\right) \\
& =\tau\left(x^{1 / 2} e_{n} h^{1 / 2} e_{n} e_{m} h^{1 / 2} e_{m} x^{1 / 2}\right)=0 .
\end{aligned}
$$

Consequently, $\varphi(x)=\sum\left(e_{n} \varphi e_{n}\right)(x)$, which shows that $\varphi$ is strictly semifinite.

(2) Let now $M$ be an arbitrary finite von Neumann algebra. Then there exists a family $\left\{z_{i}\right\}$ of non-zero central projections in $M$ such that $\sum z_{i}=1$ and each of the reduced von Neumann algebras $z_{i} M z_{i}=M z_{i}$ is $\sigma$-finite. Note that $\mathfrak{m}_{\varphi \mid M z_{i}}=\mathfrak{m}_{\varphi} z_{i}$. In fact, if $x \in \mathfrak{m}_{\varphi}^{+} z_{i}$, then $x \in M z_{i}$ and $\varphi(x)<\infty$, so that $x \in \mathfrak{m}_{\varphi \mid M z_{i}}$. If, on the other hand, $x \in\left(M z_{i}\right)_{+}$and $\varphi(x)<\infty$, then $x \in \mathfrak{m}_{\varphi}^{+} z_{i}$. Hence, for any $i, \varphi \mid M z_{i}^{+}$is semifinite. By (1), it is also strictly and densely semifinite. It follows directly from the definition of strict semifiniteness that $\varphi$ is also strictly semifinite. If now $e \in \operatorname{Proj} M$ is such that $\varphi(e)=\infty$, then for some $i$ we have $e z_{i} \neq 0$. By dense semifiniteness of $\varphi \mid\left(M z_{i}\right)_{+}$, there is a $0 \neq f \in \operatorname{Proj}\left(M z_{i}\right)$ such that $f \leq e z_{i}$ and $\varphi(f)<\infty$. We conclude that $\varphi$ is densely semifinite.

Obviously, $\varphi$ is also orthogonally semifinite, either by Proposition 2 or by Theorem $6,(1)$.

\section{Semifinite factors}

Let $M$ be a semifinite factor, and $\tau$ a faithful normal semifinite trace on $M$. If $M$ is a type $I_{\infty}$ factor, we use for $\tau$ the standard, normalized trace $\operatorname{tr}$ (i.e. $\operatorname{tr}(e)=1$ for any minimal projection $e \in M$ ). As before, we denote by $h$ the density of $\varphi$ with respect to the chosen trace $\tau$.

Proposition 8 If $M$ is a type I factor, then $\varphi$ is strictly semifinite if and only if its density $h$ with respect to the standard trace tr has pure point spectrum.

Proof If $\varphi$ is strictly semifinite, then there exists a family $\varphi_{i}$ of positive normal linear functionals $\left\{\varphi_{i}\right\}$ with mutually orthogonal supports and with $\sum \varphi_{i}=\varphi$. Then $\varphi_{i}=\operatorname{tr}\left(h_{i} \cdot\right)$ with trace class, hence also compact, densities $h_{i}$, with $\sum h_{i}=h$ strongly. Since each $h_{i}$ has pure point spectrum, so does their sum $h$. The other direction has already been proved in Theorem 6.

The next theorem is of interest only for infinite factors.

Theorem 9 If $M$ is a semifinite factor, then $\varphi$ is densely semifinite if and only if $h$ is measurable. In particular, if $M$ is a type I factor, then it is densely semifinite if and only if its density $h$ with respect to the trace tr is bounded.

Proof Assume that $h$ is not $\tau$-measurable. Let $\left(n_{k}\right)$ be an increasing sequence of natural numbers with $n_{1}=1$, such that $\tau\left(\chi_{\left[n_{k}, n_{k+1}[\right.}(h)\right) \geq 1$ (assured by non-measurability of $h$ ). Let $e_{k} \in \operatorname{Proj} M$ be such that for all $k, e_{k} \leq \chi_{\left[n_{k}, n_{k+1}[\right.}(h)$ and $\tau\left(e_{k}\right)=1$. The possibility of choosing $e_{k}$ in such a way is obvious for type $I_{\infty}$ factors, by the convention stated at the beginning of the section, and is easy to show for finite algebras, see for example [4]. For a type $I I_{\infty}$ factor, we can assume, by dense semifiniteness of $\tau$, that $\chi_{\left[n_{k}, n_{k+1}[\right.}(h)$ majorizes a finite projection in $M$ with trace $\geq 1$, and then use the result for finite algebras. In particular, since we are in a factor, all the projections $e_{k}$ are equivalent. Choose $u_{k} \in M$ so that 
$u_{k}^{*} u_{k}=e_{1}, u_{k} u_{k}^{*}=e_{k}$. Let $\left(\alpha_{k}\right)$ be a sequence of positive real numbers such that $\sum \alpha_{k}^{2}=1$ and $\sum \alpha_{k}^{2} n_{k}=+\infty$ (this is easy - just note that $n_{k} \geq k$ for all $k$ ). Put $v=\sum \alpha_{k} u_{k}$. It is easy to check that $v$ is well defined and belongs to $M$ (in fact, the sum exists in norm topology). Define $p=v v^{*}$. Since $v^{*} v=e_{1}, p \neq 0$. We shall show that if $0 \neq q \leq p$, then $\varphi(q)=\infty$. Put $g=v^{*} q v$. Then $g \leq v^{*} v=e_{1}$ and $q=v g v^{*}$. We calculate:

$$
\begin{aligned}
\varphi(q) & =\tau(h q)=\tau\left(h v g v^{*}\right) \\
& =\tau\left(v^{*} h v g\right)=\tau\left(\left(\sum_{j} \alpha_{j} u_{j}^{*}\right) h\left(\sum_{k} \alpha_{k} u_{k}\right) g\right) \\
& \geq \tau\left(\sum_{k, j} \alpha_{j} u_{j}^{*} n_{j} \alpha_{k} u_{k} g\right)=\tau\left(\sum_{k} \alpha_{k}^{2} n_{k} g\right)=+\infty .
\end{aligned}
$$

Consequently, $\varphi$ is not densely semifinite. The other direction has already been proved in Theorem 6.

\section{Counterexamples}

The examples given below show that the conditions from Theorem 6 , although sufficient, are not necessary. Although not most general possible, the examples indicate the direction of future work - finding necessary and sufficient conditions for weights to be, respectively, densely and strictly semifinite. The latter may turn out to be unfeasible, but certainly some more interesting conditions guaranteeing strict semifiniteness can be found.

Example 10 Let $N$ be a semifinite von Neumann algebra with faithful normal semifinite trace $\tau_{N}$, and let $K$ be a $\sigma$-finite non-atomic commutative von Neumann algebra. Put $M=$ $N \bar{\otimes} K$ and $\tau=\tau_{N} \otimes \mu$, where $\mu$ corresponds to the Lebesgue measure in the isomorphism between $K$ and $L^{\infty}([0,1], L e b)$. Then there exists a strictly semifinite weight on $M$ whose density with respect to $\tau$ does not have a pure point spectrum.

Proof Let $m_{f}$ be a multiplication operator on $L^{2}([0,1], L e b)$ with $f(t)=t$ for $t \in[0,1]$. Put $h=1 \otimes m_{f}$. It is clear that the spectrum of $h$ is not a pure point one. On the other hand, the weight $\varphi$ with density $h$ with respect to $\tau$ is, in fact, a trace, hence it is strictly semifinite by Proposition 3 .

Example 11 Let $M$ be a semifinite von Neumann algebra and $\tau$ an infinite faithful normal semifinite trace on $M$. Suppose that the restriction of $\tau$ to $\mathcal{Z}(M)$ is semifinite (which implies that the center of $M$ is infinite dimensional). Then there exists a densely semifinite weight $\varphi$ on $M$ such that its density with respect to $\tau$ is non- $\tau$-measurable.

Proof By Proposition 3, the restriction of $\tau$ to (the positive part of) the center of $M$ is densely semifinite, hence there exists a family $\left\{z_{i}\right\}$ of non-zero projections from $\mathcal{Z}(M)$ such that $\tau\left(z_{i}\right)<+\infty$ for all $i$, and $\sum z_{i}=1$. Since $\tau(1)=+\infty$, the family is infinite, and we can suppose (grouping the projections if necessary) that the family is countable (indexed by $k \in \mathbb{N}$ ), and that $\tau\left(z_{k}\right) \geq 1$ for all $k$. Let $h=\sum k z_{k}$. Then $h$ is well and densely defined, and obviously affiliated with $M$. Note that $h$ is not $\tau$-measurable: $\tau\left(\chi_{[n, \infty[}(h)=\tau\left(\sum_{k \geq n} z_{k}\right)=+\infty\right.$. On the other hand, $h$ is bounded, hence measurable, on each $M z_{k}$, which means that the weight $\varphi$ given by $\varphi=\tau(h \cdot)$ is densely semifinite on each $M z_{k}$, by Theorem 6. Consequently, $\varphi$ is densely semifinite (see the second part of the 
proof of Proposition 7). Thus, we have a densely defined semifinite weight (even a trace) with density which is not $\tau$-measurable.

Any von Neumann algebra can be equipped with a faithful normal strictly semifinite weight. This result is both classical and easy. It is not a priori evident that there exist nonstrictly semifinite weights on type $I I_{\infty}$ or type $I I I$ algebras, but there are two non-trivial examples guaranteeing their existence. First, Haagerup [5] proved that there is a densely defined positive operator $h$ affiliated with type $I I_{\infty}$ von Neumann algebra such that the von Neumann algebra $\{h\}^{\prime}$ consisting of operators commuting with $h$ is of type III. Note that the algebra is in fact a centralizer of the weight $\varphi=\tau(h \cdot)$, which means that the weight cannot be strictly semifinite (otherwise its restriction to the type III algebra would be a semifinite trace). Secondly, Herman and Takesaki [6] showed that one can built a faithful normal semifinite weight on a type III algebra with trivial centralizer. Again, such a weight cannot be strictly semifinite.

The situation concerning dense and orthogonal semifiniteness in type III algebras is a mystery. We do not know if there exist semifinite weights that are not orthogonally semifinite. We do not know whether there exist densely semifinite weights in factors of type III, and we do not know whether there exist weights that are not densely semifinite in such factors. The simple nature of the definitions suggests that clearing up the circumstances can be useful when proving theorems in type III algebras.

\section{References}

1. Combes, F.: Poids sur une *-algèbre. J. Math. Pures Appl. 47, 57-100 (1968)

2. Combes, F.: Poids associés à une algèbre hilbertienne à gauche. Compositio Math. 23, 49-77 (1971)

3. Combes, F.: Poids et espérances conditionelles dans les algèbre de von Neumann. Bull. Soc. Math. France 99, 73-112 (1971)

4. Goldstein, S., Paszkiewicz, A.: Comparison of states and Darboux-type properties in von Neumann algebras. Math. Scand. 63, 220-232 (1988)

5. Haagerup, U.: An example of a weight with type III centralizer. Proc. Amer. Math. Soc. 62, 278-280 (1977)

6. Herman, R.H., Takesaki, M.: States and automorphism groups of operator algebras. Comm. Math. Phys. 19, 142-160 (1970)

7. Strătilă, Ş.: Modular theory in operator algebras. Abacus Press, Tunbridge Wells (1981)

8. Takesaki, M.: Theory of operator algebras I, Reprint of the first (1979) edition (2002)

9. Takesaki, M.: Theory of operator algebras II. Springer-Verlag, Berlin (2003) 